\title{
Feedback Control of the Arabidopsis Hypersensitive Response
}

\author{
Chu Zhang, ${ }^{1}$ Annie Tang Gutsche, ${ }^{2}$ and Allan D. Shapiro ${ }^{1}$ \\ 'Department of Plant and Soil Sciences, Delaware Agricultural Experiment Station, College of Agriculture and Natural \\ Resources, University of Delaware, Newark, DE 19716-2170, U.S.A.; ${ }^{2}$ DuPont Central Research and Development, \\ Experimental Station, Wilmington, DE 19880-0228, U.S.A.
}

Submitted 29 August 2003. Accepted 17 November 2003.

\begin{abstract}
The plant hypersensitive response (HR) to avirulent bacterial pathogens results from programmed cell death of plant cells in the infected region. Ion leakage and changes in signaling components associated with HR progression were measured. These studies compared Arabidopsis mutants affecting feedback loops with wild-type plants, with timepoints taken hourly. In response to Pseudomonas syringae pv. tomato DC3000॰avrB, npr1-2 mutant plants showed increased ion leakage relative to wild-type plants. Hydrogen peroxide accumulation was similar to that in wild type, but salicylic acid accumulation was reduced at some timepoints. With DC3000•avrRpt2, similar trends were seen. In response to $\mathrm{DC3000}$ avrB, ndr1-1 mutant plants showed more ion leakage than wild-type or npr1-2 plants. Hydrogen peroxide accumulation was delayed by approximately $1 \mathrm{~h}$ and reached half the level seen with wild-type plants. Salicylic acid accumulation was similar to npr1-2 mutant plants. With DC3000•avrRpt2, ndr1-1 mutant plants showed no ion leakage, no hydrogen peroxide accumulation, and minimal salicylic acid accumulation. Results with a ndr1-1 and npr1-2 double mutant were similar to ndr1-1. A model consistent with these data is presented, in which one positive and two negative regulatory circuits control HR progression. Understanding this circuitry will facilitate HR manipulation for enhanced disease resistance.
\end{abstract}

The hypersensitive response (HR) is a macroscopic collapse of infected plant leaves that is highly correlated with gene-forgene disease resistance. This leaf collapse reflects programmed cell death (PCD) of most plant cells in the leaf. Leakage of ions and water from these cells followed by transpirational water loss produces the macroscopic leaf collapse (Goodman and Novacky 1994). The HR to avirulent bacteria is elicited by type III secretion-dependent delivery of bacterial avirulence (avr) gene products into plant leaf cells (Casper-Lindley et al. 2002; Gopalan et al. 1996; Leister et al. 1996; Pirhonen et al. 1996; Wu et al. 2003). PCD occurs only in plants carrying a resistance $(R)$ gene that matches the bacterial $a v r$ gene. With the Arabidopsis thaliana $R$ genes RPS 2 and $R P M 1$, molecular recognition of the cognate avr genes (avrRpt2 and avrB/avrRpm1, respectively) occurs at the cytoplasmic face of the plasma membrane and also involves other Arabidopsis proteins (Axtell and Staskawicz 2003; Mackey et al. 2002, 2003).

The signaling pathway leading to PCD is at present unclear. Evidence has been presented for importance of ion fluxes

Corresponding author: A. D. Shapiro; E-mail: ashapiro@udel.edu
(Atkinson et al. 1986, 1990; Baker et al. 1987; Clough et al. 2000; Grant et al. 2000; Xu and Heath 1998), nitric oxide production (Clarke et al. 2000; Delledonne et al. 1998; Durner et al. 1998), caspase-like protease activity (del Pozo and Lam 1998; Lam and del Pozo 2000), and changes to mitochondria (Lam et al. 2001). An early oxidative burst correlated with the HR was proposed to be the major trigger of PCD, perhaps in combination with other signals (Delledonne et al. 2001; Glazener et al. 1996; Mazel and Levine 2001; McDonald et al. 2002; Mur et al. 2000; Orlandi et al. 1992). Indeed, mutants that accumulate high levels of reactive oxygen show lesion mimic phenotypes that resemble the HR (Hu et al. 1998; Jabs et al. 1996; Takahashi et al. 1999), and knockouts of NADPH oxidase genes responsible for reactive oxygen production show altered HRs (Torres et al. 2002).

In the Arabidopsis HR, however, high-level production of reactive oxygen does not occur until after the first PCD events and is thus likely a consequence rather than a cause of PCD (Shapiro and Zhang 2001; Zhang et al. 2003). Similar conclusions were reached in studies of the cowpea HR to avirulent cowpea rust fungus (Heath 1998). We have also proven that nitric oxide is not an early signaling intermediate by showing that it does not accumulate prior to the first PCD events (Zhang et al. 2003). Nevertheless, these studies do not rule out a role for either reactive oxygen or nitric oxide as a contributory cause of PCD late in HR progression.

Salicylic acid (SA) accumulation can contribute to the HR but is not essential in all cases (Kachroo et al. 2000; Mur et al. 1997; Rate et al. 1999; Shapiro and Zhang 2001). SA was postulated, based on cell culture studies, to act in "agonist-dependent gain control." By itself, SA did not kill plant cells. However, it potentiated weak avr gene-dependent signals, leading to higher levels of cell death (Shirasu et al. 1997).

We have proven that this gain-control mechanism is operative in the Arabidopsis HR in a study focusing on the $n d r 1-1$ mutant (Shapiro and Zhang 2001). The $n d r l-1$ mutation prevents the HR to bacteria carrying avrRpt2 (Century et al. $1995,1997)$ except at extremely high levels of inoculum (Shapiro 2000). The ndrl-1 mutation does not prevent the HR to bacteria carrying any of four other $a v r$ genes (Century et al. 1995; Shapiro 2000). The $n d r l$ mutation impairs reactive oxygen-dependent induction of SA biosynthesis (Shapiro and Zhang 2001). The sid2 mutant, which blocks one of the two SA biosynthetic pathways (Wildermuth et al. 2001), also shows no HR to bacteria carrying avrRpt2, when assayed under standard conditions (Zhang and Shapiro 2002). This mutant does show a HR when very high levels of inoculum are used (Nawrath and Métraux 1999). We postulated that impairment of SA accumulation blocked only the avrRpt2- 
elicited HR because the "triggering" of this HR was weaker than the triggering of other HRs. HRs triggered by "stronger" signals would not require SA potentiation. The basis for our conclusion was the more gradual progression of the avrRpt2elicited HR (Grant et al. 2000; Ritter and Dangl 1996; Shapiro and Zhang 2001).

A molecular basis for this weaker triggering has recently been proposed. Triggering of the HR to avrRpt 2 requires nearly quantitative depletion of RIN4 levels to relieve stoichiometric inhibition of RPS2 activity (Mackey et al. 2003). Triggering of the HR to $a v r B$ or avrRpml is more rapid, possibly because it appears to require activation of only a fraction of the cellular RPM1 pool (Mackey et al. 2002). Consistent with this explanation, moderate overexpression of RPS2 greatly sped the avrRpt2-dependent HR (Axtell and Staskawicz 2003) and overexpression of RIN4 blocked this HR (Mackey et al. 2003).

Agonist-dependent gain control provides a mechanistic explanation for SA-dependent positive feedback on PCD (Shapiro and Zhang 2001; Weymann et al. 1995). SA synthesized by either the earliest cells to commit to PCD, by surrounding cells, or both potentiates triggering of PCD in cells that die later in the HR. However, the HR also appears to be controlled by negative feedback. The greater extent of leaf collapse seen in $n d r l$ mutants as compared with wild-type plants in response to $a v r B$, avrRpm1, avrPphB, or avrRps4 (Century et al. 1995, 1997; Shapiro 2000) was taken as evidence of SAdependent negative feedback controlling the extent of cell death (Shapiro and Zhang 2001). Indeed, treatment with exogenous SA can block the HR (Devadas and Raina 2002). Quantitative assay of the HR in $n p r l$ mutants showed a higher level of ion leakage measured $15 \mathrm{~h}$ postinoculation with bacteria carrying either avrRpt 2 or avrRpml (Rate and Greenberg 2001). This result suggested that negative feedback was NPRl-dependent. NPRI-dependent negative feedback has also been suggested by experiments showing enhancements in SA levels in $n p r l$ mutant plants 1 to 2 days postinoculation with Pseudomonas syringae pv. tomato DC3000 (hereafter, DC3000) carrying avrRpt2 (Delaney et al. 1995; Shah et al. 1997). The relationships between these feedback loops were not clear from these studies.

We have recently reported construction of an $n d r l-1$ and nprl-2 double mutant. This double mutant was used to deline- ate signaling pathways leading to systemic acquired resistance and pathogenesis-related gene induction on the basis of largely additive effects of the two mutations (Zhang and Shapiro 2002). In the present study, the single and double mutant lines were used to investigate control over the HR. Quantitative analyses of ion leakage, hydrogen peroxide accumulation, and SA accumulation were performed under identical conditions. The data support a model in which HR progression is governed by three interacting feedback loops. This model explains the dynamic changes in signaling components resulting from infection with avirulent bacteria.

\section{RESULTS}

\section{The ndr1-1 mutation is epistatic}

to $n p r$ 1-2 with respect to macroscopic HR phenotypes.

As a first step towards understanding control over the HR, single and double-mutant lines were compared with respect to macroscopic HR phenotypes. The data is presented in Table 1. Columbia plants showed the expected HR when observed approximately $20 \mathrm{~h}$ postinoculation with DC3000 carrying avrB, avrRpt2, avrRpm1, or avrPphB. No HR was seen to DC3000•empty vector. Results with nprl-2 plants were identical to those with Columbia. Consistent with results reported in the literature (Century et al. 1995, 1997), $n d r l-1$ plants did not give a HR to DC3000•avrRpt2. However, they showed an exaggerated HR to DC3000•avrB, DC3000•avrRpml, and DC3000•avrPphB. The double-mutant line showed identical results to those obtained with $n d r l-1$. The completely consistent results obtained with 15 replicate leaves for each combination of plant line and bacterial strain indicates full epistasis in this assay.

In an attempt to identify subtle phenotypic differences, these experiments were repeated with different bacterial host strain backgrounds. A different pathogen strain (Pseudomonas syringae pv. maculicola strain ES4326) and two strains that are not pathogens of Arabidopsis (Pseudomonas syringae pv. glycinea Race 5 and Pseudomonas syringae pv. phaseolicola strain 3121) carrying each of the $a v r$ genes were employed. Nonpathogenic strains were used at four different levels of inoculum (discussed below). In all cases, nprl-2 plants were identical to Columbia, and the double-mutant plants were identical to ndrl-1 (data not shown). HR phenotypes elicited by bacteria

Table 1. Macroscopic hypersensitive response (HR) determinations indicate that $n d r 1-1$ is epistatic to $n p r l-2$

\begin{tabular}{lccccc}
\hline Plant lines $^{\mathbf{a}}$ & Empty vector $^{\mathbf{b}}$ & avrB & avrRpt2 & avrRpmI & avrPphB \\
\hline Columbia & - & + & + & + & + \\
$n$ ndrl-1 & - & ++ & - & + & ++ \\
$n p r l-2$ & - & + & + & + & + \\
ndrl-1/nprl-2 & - & ++ & - & ++ \\
\hline
\end{tabular}

${ }^{a}$ The indicated plant lines were inoculated with $P$. syringae pv. tomato DC3000 carrying the indicated avirulence gene on the pVSP61 vector at concentrations of $2 \times 10^{7}$ per ml. With each combination of plant line and bacterial strain, 15 leaves were assessed approximately $20 \mathrm{~h}$ postinoculation. Identical results were obtained with all 15 leaves for each combination.

b - indicates no HR; + indicates that some degree of leaf collapse was noted; and ++ indicates exaggerated leaf collapse relative to wild type.

Table 2. Kinetic parameter values from ion leakage data fit to sigmoid functions ${ }^{\mathrm{a}}$

\begin{tabular}{|c|c|c|c|c|c|}
\hline & \multicolumn{3}{|c|}{ DC3000•avrB } & \multicolumn{2}{|c|}{ DC3000•avrRpt2 } \\
\hline & Columbia & npr1-2 & ndr1-1 & Columbia & nprl-2 \\
\hline Amplitude & $0.34(0.01)$ & $0.44(0.02)$ & $0.54(0.02)$ & $0.35(0.02)$ & $0.45(0.03)$ \\
\hline Baseline & $0.08(0.00)$ & $0.10(0.01)$ & $0.12(0.01)$ & $0.09(0.01)$ & $0.08(0.02)$ \\
\hline Midpoint & $5.1(0.1)$ & $5.1(0.1)$ & $5.4(0.1)$ & $6.3(0.2)$ & $6.3(0.3)$ \\
\hline Slope & $1.5(0.1)$ & $1.7(0.2)$ & $1.8(0.2)$ & $1.0(0.2)$ & $1.2(0.3)$ \\
\hline
\end{tabular}

${ }^{a}$ Data was fit to sigmoid functions. The values for each of the four kinetic parameters fit by the software are shown. Residual errors of the fits are shown in parentheses. Differences between mutants inoculated with the same bacterial strain were compared using Student's $t$ tests. Differences in final amplitude of the curves relative to initial baselines were highly significant $(P<0.01$ for all pairwise comparisons). 
carrying avrRps4 on Columbia can be assessed reproducibly only by using high levels of inoculum and a nonpathogenic strain background (Shapiro 2000). In these experiments, $n p r l-2$ resembled Columbia. The double mutant showed an exaggerated HR that was similar to that shown by ndrl-1 (data not shown). There were thus no differences in HR between $n d r 1-1$ and the double mutant that could be assessed by qualitative observation of leaf collapse.

\section{Quantitative analysis of HR progression.}

A time course study of ion leakage associated with HR progression was next undertaken to determine when and to what extent these mutants show differences in HR control. These assays measured loss of ions caused by plasma membrane damage characteristic of plant PCD events associated with the HR (Goodman and Novacky 1994). Samples at individual timepoints consisted of 10 leaf disks taken from a single plant. Each timepoint used a different plant. Each sample was washed to minimize contribution from ions leaking from the cut edges. The samples were vacuum infiltrated to assure uniform contact of leaf cells with the purified water. Conductivity was then read $10 \mathrm{~min}$ after the infiltration procedure. Conducting the assay in this fashion assured measurements were made approximately $15 \mathrm{~min}$ after sampling. This time interval was minimized in order to most closely approximate in vivo conditions. Measurements were normalized to total ions releasable by boiling the samples. Each time course experiment was repeated at least five times, and averaged data is shown. Infections with DC3000•empty vector elicited no discernible increase in conductivity over the timepoints sampled in any of the experiments (data not shown).

Figure $1 \mathrm{~A}$ and $\mathrm{B}$ shows ion leakage associated with HR progression in response to DC3000•avrRpt2. Consistent with the macroscopic HR phenotypes, $n d r 1-1$ and $n d r 1-1 / n p r 1-2$ plants showed no increase in conductivity over the timepoints sampled. Columbia and nprl-2 plants showed sigmoidal kinetics of increase in conductivity with time. Nonlinear, least squares fitting of an equation for sigmoidal kinetics (discussed below) was used for analysis of the data. This treatment allowed separate examination of curve amplitude, baseline, midpoint, and slope in that the software fit all four parameters. A typical curve associated with this analysis is displayed in Figure 1A. Values of these kinetic parameters are listed in Table 2. The amplitude from experiments with nprl-2 plants was significantly higher than that calculated from Columbia data (Student's $t$ test, $P<0.01$ ). This difference was consistent with the published conclusion that nprl mutations impair negative feedback on the HR (Rate and Greenberg 2001). The other parameters did not show significant differences from Columbia.

Figure 1C shows ion leakage in response to DC3000•avrB. As in our previous work (Shapiro and Zhang 2001; Zhang and Shapiro 2002), we chose $a v r B$ as a representative of the class of $a v r$ genes that can elicit a macroscopic HR on $n d r 1-1$ plants under standard conditions of assay. Kinetic parameters are presented in Table 2. As with DC3000•avrRpt2, experiments with nprl-2 showed significantly higher amplitudes than those with Columbia. Experiments with $n d r l-1$ plants showed significantly higher amplitudes than those with either Columbia or nprl-2 (Student's $t$ tests, $P<0.01$ for all pair-wise comparisons). We conclude that negative feedback was impaired in both single mutant lines and that $n d r l-1$ showed greater impairment than $n p r 1-2$. Differences in other kinetic parameters were minor and most were not statistically significant.

Kinetic parameters associated with HR progression in the double mutant could not be compared with the other data, because the curve did not saturate prior to the timepoint at which macroscopic collapse precluded further measurements.
A
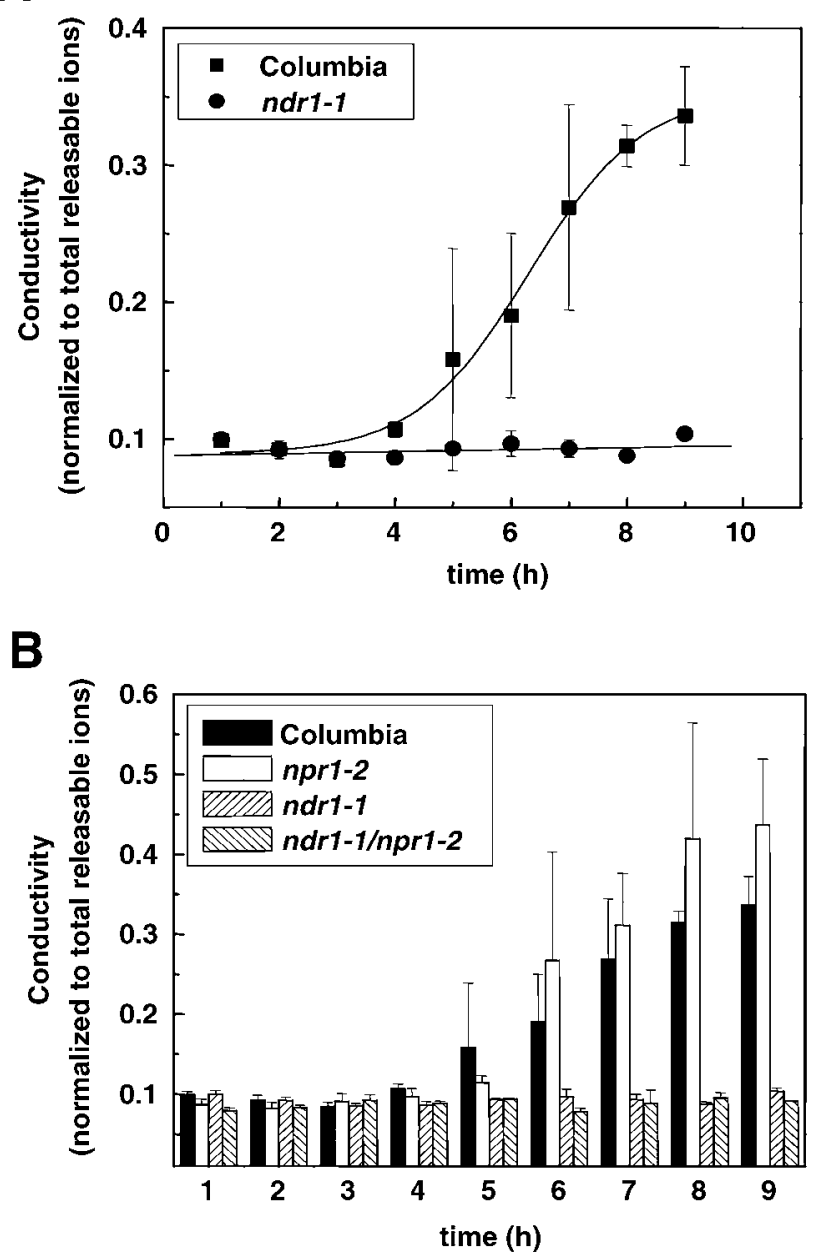

C

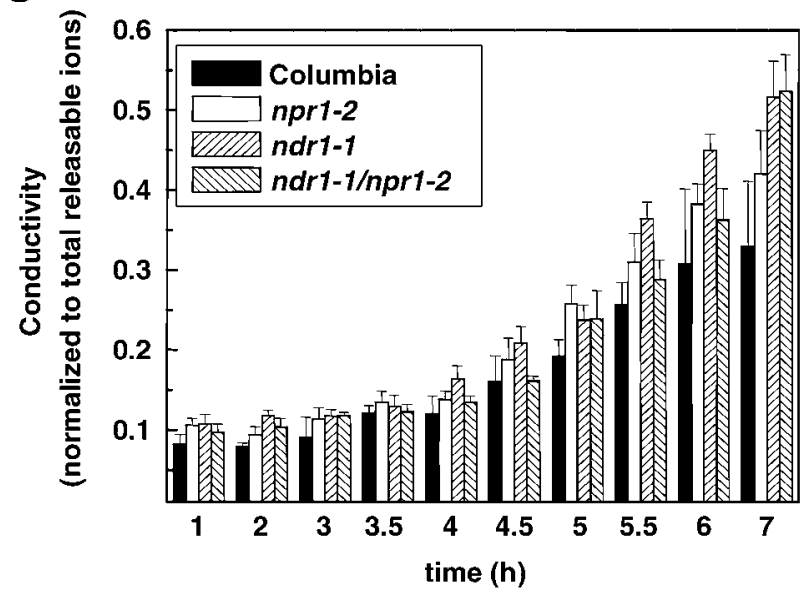

Fig. 1. Quantitative analysis of ion leakage correlated with hypersensitive response progression. A, Columbia or $n d r 1-1$ plants were infected with 2 $\times 10^{7}$ DC3000•avrRpt2 per $\mathrm{ml}$ and were sampled at the indicated timepoints. Conductivity of water containing ions leaked from leaf samples was measured and normalized to total ions releasable by boiling. Results from five separate experiments were averaged. Error bars represent standard deviations of means of data from single timepoints. Columbia data were fit to a sigmoid function $(R=0.996)$. Data from ndr1-1 plants were fit to a straight line. B, Infections, sampling, conductivity measurements, and treatment of data were as in A. The plant lines used are indicated. $\mathbf{C}$, The indicated plant lines were infected with 2 $\times 10^{7} \mathrm{DC} 3000 \bullet a v r B$ per $\mathrm{ml}$ and were sampled at the indicated timepoints. Timepoints were not taken after $7 \mathrm{~h}$ postinoculation because onset of leaf collapse precluded further sampling. Infections, sampling, conductivity measurements, and treatment of data were as in A. 
However, Figure 1C shows that the normalized conductivity readings seen at the 7-h timepoint were similar to those of $n d r 1-1$. The high final reading is consistent with the exaggerated HR shown by both $n d r l-1$ and the double mutant in qualitative, macroscopic assays.

\section{$\mathrm{H}_{2} \mathrm{O}_{2}$ accumulation during $\mathrm{HR}$ progression.}

We have previously published measurements of $\mathrm{H}_{2} \mathrm{O}_{2}$ levels during progression of the HR (Shapiro and Zhang 2001). However, the assay for $\mathrm{H}_{2} \mathrm{O}_{2}$ accumulation used had a low signal-to-noise ratio. A Polaroid camera was used to capture fluorescence increases that report oxidation of 2',7'-dichlorodihydrofluorescein (DCFH) by $\mathrm{H}_{2} \mathrm{O}_{2}$ (Wolfe et al. 2000). This method could not filter out background red fluorescence due to chlorophyll. We have modified the previous assay by using an epifluorescence microscope equipped with a wide-field lens to image and quantify fluorescence (discussed below). Only the green DCF fluorescence was captured. Red chlorophyll fluorescence was filtered out. In Figure 2, the left half-leaf in the image on the left was inoculated with DC3000•empty vector $7 \mathrm{~h}$ prior to sampling. Only localized, faint fluorescence (e.g., surrounding the needle hole) was evident. These areas would have been avoided during quantitation. By contrast, the image on the right shows very strong green fluorescence. The left half-leaf in this image was inoculated with DC3000•avrB $7 \mathrm{~h}$ prior to sampling.

The excellent signal-to-noise thus obtained allowed quantitation of the response (discussed below). The data is presented in Figure 3. Infection with DC3000•empty vector elicited no discernible increases in $\mathrm{H}_{2} \mathrm{O}_{2}$ accumulation in any of the four plant lines (Fig. 3B, dotted lines). In response to infection with DC3000•avrRpt2 under conditions identical to those used in quantitation of HR progression, Columbia and nprl-2 plants showed a gradual increase in $\mathrm{H}_{2} \mathrm{O}_{2}$ accumulation starting $5 \mathrm{~h}$ postinoculation (Fig. 3A). The slightly higher level of $\mathrm{H}_{2} \mathrm{O}_{2}$ accumulation displayed by Columbia plants $3 \mathrm{~h}$ postinoculation is not likely to be significant, as it is comparable to the level shown in response to DC3000•empty vector (Fig. 3B). Differences between Columbia and $n p r l-2$ at later timepoints were small. By contrast, $n d r l-1$ and double-mutant plants showed no discernible increases in $\mathrm{H}_{2} \mathrm{O}_{2}$ accumulation over the timepoints sampled. These results are correlated with the absence of a HR (Table 1 and Fig. 1).

In response to DC3000•avrB (Fig. 3B), Columbia and nprl-2 plants showed $\mathrm{H}_{2} \mathrm{O}_{2}$ accumulation beginning approximately $1 \mathrm{~h}$ earlier than with DC3000•avrRpt2. The rates of

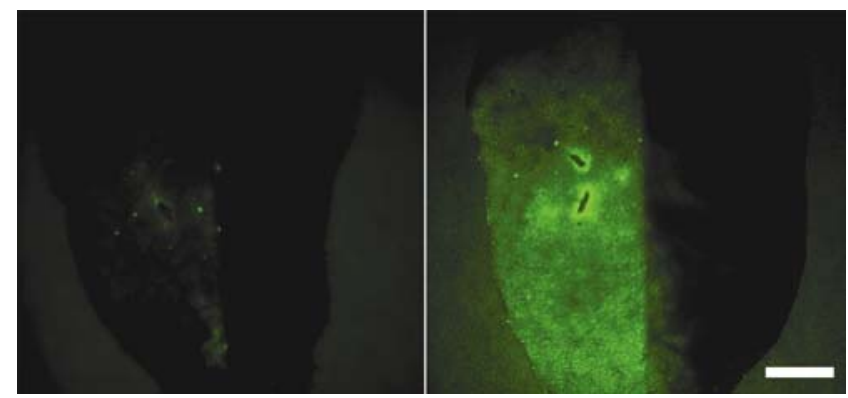

Fig. 2. Fluorescence-based measurement of $\mathrm{H}_{2} \mathrm{O}_{2}$ accumulation in infected Arabidopsis leaves. The left halves of the two leaves pictured were inoculated with $2 \times 10^{7} \mathrm{DC} 3000 \bullet$ empty vector (left) or DC3000•avrB (right) per ml. 2',7'-Dichlorodihydrofluorescein-diacetate was introduced into leaves $15 \mathrm{~min}$ prior to harvest for microscopic imaging of fluorescence. The area of low level fluorescence surrounding the needle hole in the image on the left would have been avoided in quantitation of fluorescence. Images were taken $7 \mathrm{~h}$ postinoculation. Scale bar $=0.25 \mathrm{~cm}$ (both panels at same level of magnification). increase were faster, and the final curve amplitudes were somewhat higher. Increases in $\mathrm{H}_{2} \mathrm{O}_{2}$ accumulation were also seen with ndrl-1 and double-mutant plants. The responses in these two lines were very similar to each other. However, the responses commenced approximately $1 \mathrm{~h}$ later than with $\mathrm{Co}-$ lumbia and nprl-2 plants. Furthermore, the maximal change observed in average pixel intensity relative to baseline in $n d r l-1$ and double-mutant plants was approximately half that seen with Columbia and nprl-2 plants. Thus, in response to DC3000•avrB, the $n d r 1-1$ mutation affected both the time of

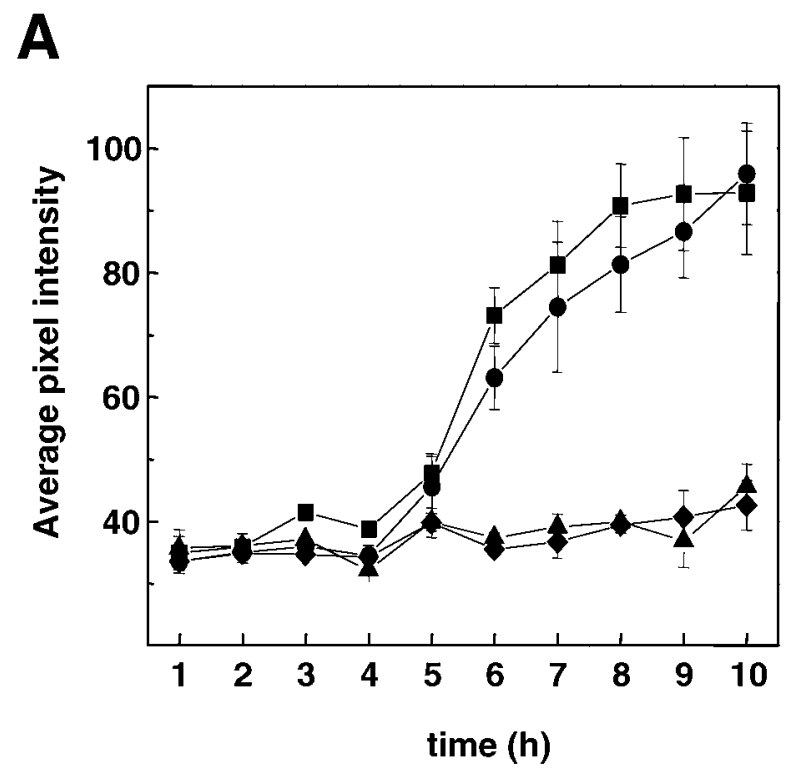

B

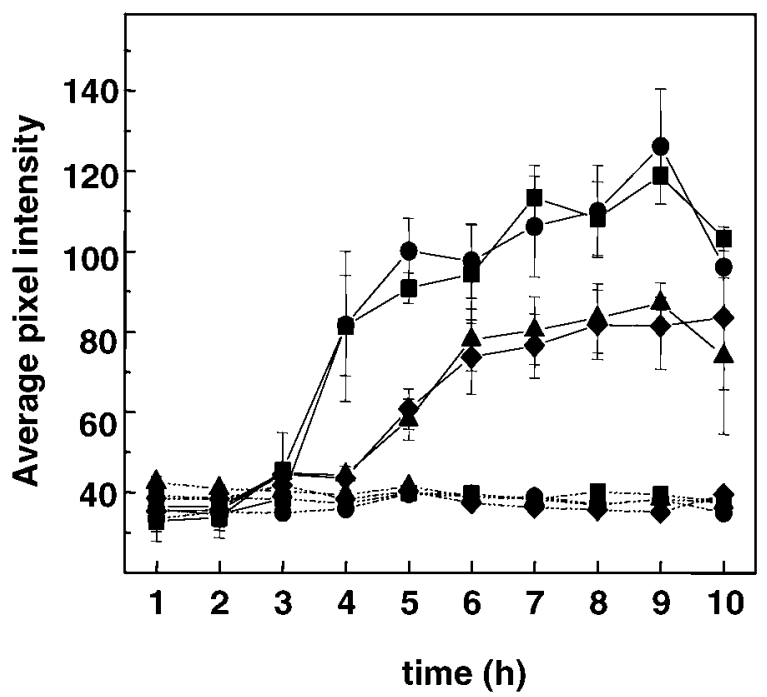

Fig. 3. Kinetics of $\mathrm{H}_{2} \mathrm{O}_{2}$ accumulation during the hypersensitive response A, Columbia wild-type (squares), nprl-2 (circles), ndrl-1 (triangles), or ndr1-1/npr1-2 (diamonds) plants were infected with $2 \times 10^{7}$ DC3000•avrRpt2 per ml. DCFH-DA was introduced into leaves through the original needle holes $15 \mathrm{~min}$ prior to the indicated timepoints. Pixel intensity was measured in three areas per leaf. The entire experiment was repeated three times using the identical microscope settings. Individual data points represent means of the three averaged pixel-intensity measurements (nine measurements total per timepoint for each plant line). Error bars represent standard errors of these means. B, The same plant lines as in A were infected with $2 \times 10^{7} \mathrm{DC} 3000 \bullet a v r B$ (solid lines) or DC3000•empty vector (dashed lines) per ml. Fluorescence measurements and data treatment were as in A. 
onset and final extent of $\mathrm{H}_{2} \mathrm{O}_{2}$ accumulation, whereas the nprl-2 mutation did not show discernible effects.

\section{SA accumulation during HR progression.}

Time course studies of SA accumulation under identical conditions to those used to measure HR progression and
$\mathrm{H}_{2} \mathrm{O}_{2}$ accumulation were next undertaken. We have recently established a capillary electrophoresis-based protocol for SA quantitation that requires lower amounts of infected leaf tissue than extant high-performance liquid chromatographybased protocols do (Shapiro and Gutsche 2003). This advance made possible a time course study with sufficient

A

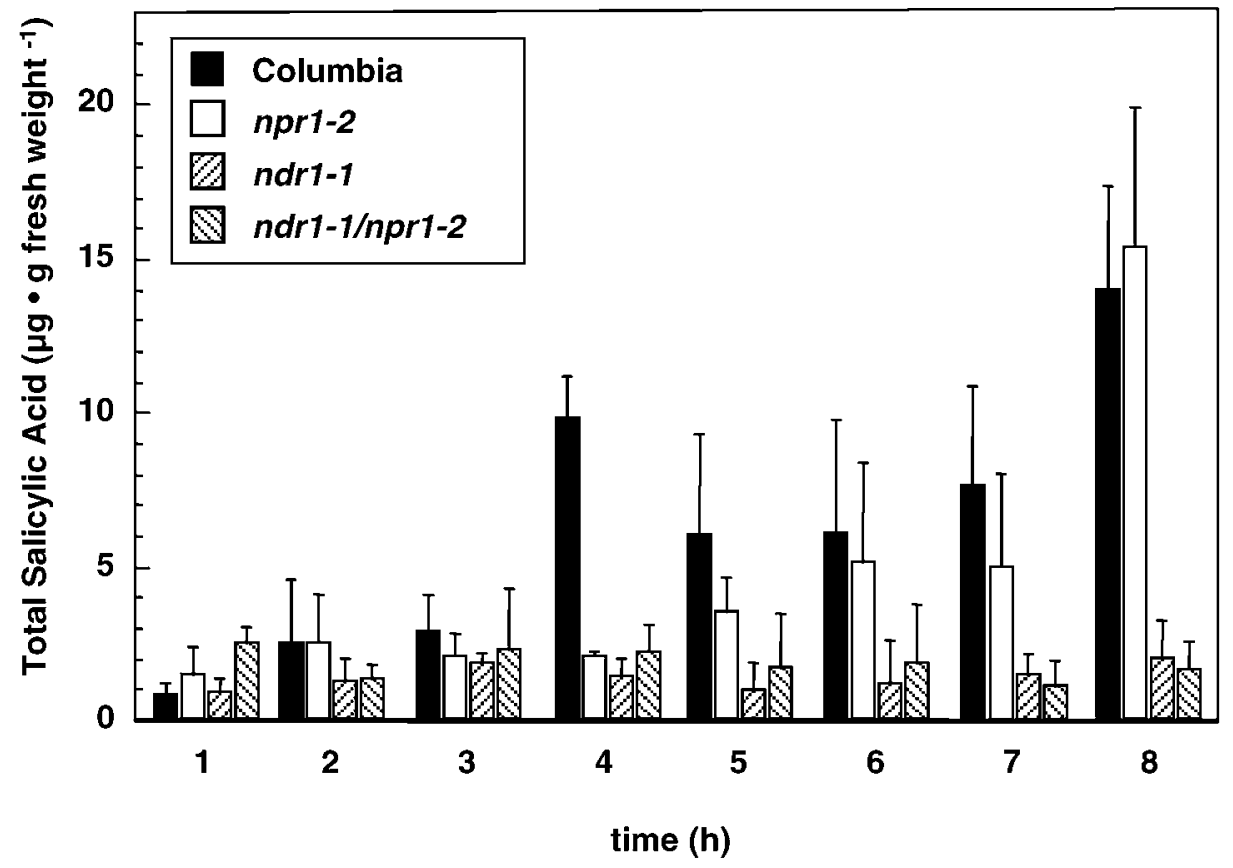

B

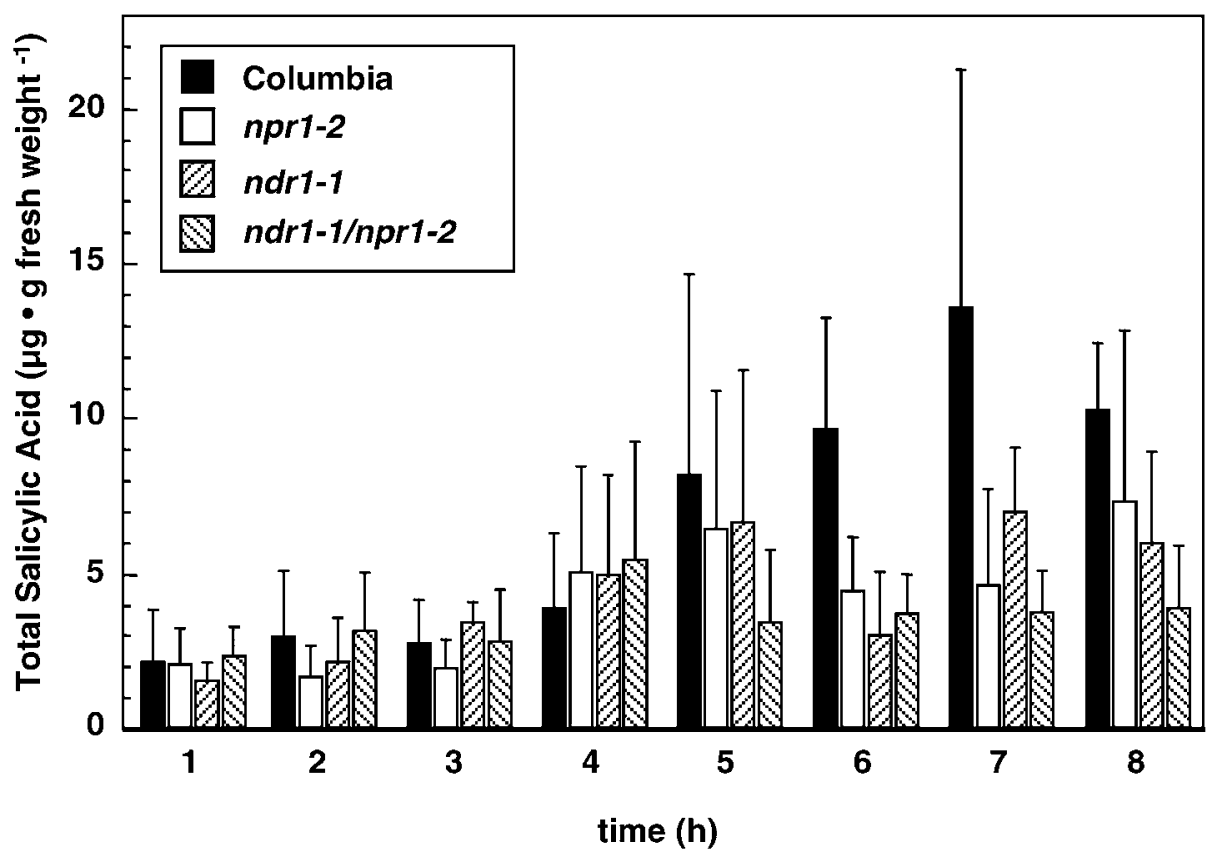

Fig. 4. Kinetics of salicylic acid (SA) accumulation during the hypersensitive response. A, The indicated plant lines were infected with $2 \times 10^{7}$ DC3000•avrRpt2 per ml and were sampled at the indicated timepoints. Fractions enriched in phenolics were prepared and subjected to capillary electrophoresis-based quantitation of total SA levels. The entire experiment was repeated four times. Error bars represent standard deviations of means of these four replicate determinations. B, The same plant lines as in A were infected with $2 \times 10^{7} \mathrm{DC} 3000 \bullet a v r B$ per ml and were sampled once per hour for the first $8 \mathrm{~h}$ postinoculation. Quantitation of total SA levels was as in A. Temporal asynchrony was seen between replicate experiments. Timing of large increases in SA accumulation in some samples was offset by approximately $1 \mathrm{~h}$ relative to replicate samples. Nonetheless, simple averaging was used to show the best determinations of levels of total SA accumulation to be expected at each timepoint, resulting in the large error bars. 
experimental replicates using all four plant lines and all three bacterial strains used above (all experiments performed in quadruplicate). The data is presented in Figure 4. Infection with DC3000•empty vector elicited no discernible increases in SA accumulation in any of the four plant lines over the timepoints sampled (data not shown). In response to DC3000•avrRpt2, Columbia plants showed modest increases in SA accumulation over the first $3 \mathrm{~h}$. The levels did not peak and then decrease during this early period, as they did when a lower level of inoculum was employed (Shapiro and Gutsche 2003) or a nonhost HR was studied in tobacco (Mur et al. 2000). The level of SA accumulation seen $4 \mathrm{~h}$ postinoculation was dramatically higher than that seen at the earlier timepoints. Levels further increased (Student's $t$ test, $P<0.05)$ to $14 \mu \mathrm{g}$ total SA per $\mathrm{g}$ of fresh weight by $8 \mathrm{~h}$ postinoculation.

All three mutant lines showed striking differences from Columbia in this assay. The largest difference between nprl-2 and Columbia was seen at the 4-h timepoint. With ndrl-1 and double-mutant plants, although small increases in SA levels were seen relative to uninoculated plants $(\leq 1 \mu \mathrm{g}$ per $\mathrm{g}$ of fresh weight in uninoculated plants; data not shown), the major increases in SA accumulation seen with Columbia and nprl-2 plants did not happen.

In response to DC3000• $a v r B$, the effect of the mutations on SA accumulation was much less pronounced than with DC3000•avrRpt2. Columbia plants showed similar amplitudes of SA accumulation to those seen with DC3000•avrRpt2. However, a much greater degree of asynchrony was seen between the four replicates of the experiments than was seen with DC3000•avrRpt2. Most of the variation was in the timing rather than in the amplitude of the responses. With two of the replicates, increases in SA accumulation trailed the other two replicates by approximately $1 \mathrm{~h}$. Simple averaging of replicate data gave rise to large error bars, especially at points where a minimum in some replicates was averaged with points from ascending trajectories in others. Nonetheless, all three mutants showed significantly less accumulation than Columbia plants did at later timepoints (Student's $t$ tests, $P<0.05$ for comparisons using data from 6- or 7-h timepoints).

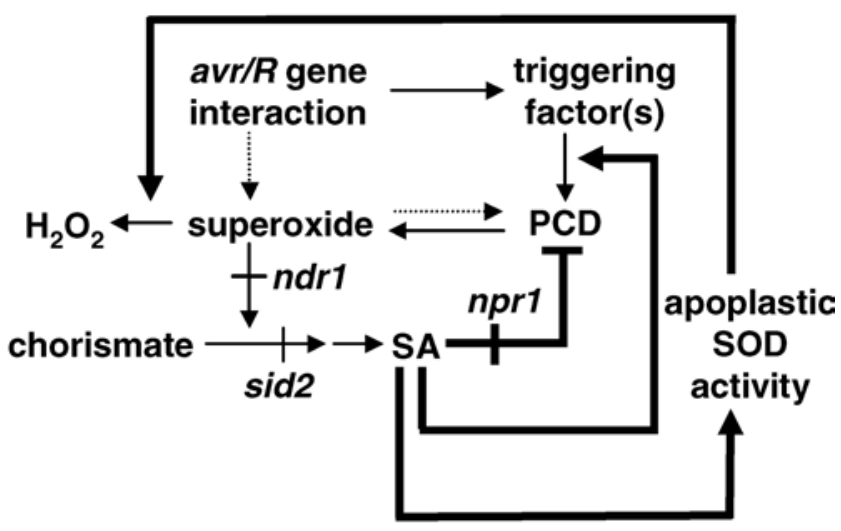

Fig. 5. Feedback regulation of the Arabidopsis hypersensitive response (HR). A working hypothesis describing integration of the major pathways of information and metabolic flux affecting HR progression is presented. Arrows designate pathways of metabolic flux or information flow. Dashed lines indicate pathways that are comparatively minor in wild-type Columbia Arabidopsis responding to avirulent bacteria. Heavier weight lines are used to show three feedback loops. Signaling blocks in Arabidopsis mutants are indicated by a slash adjacent to the mutant name. Some aspects of salicylic acid biosynthesis and its regulation have been omitted for clarity of presentation.

\section{DISCUSSION}

A model that explains the data presented in this paper and previously published data is presented in Figure 5. Evidence for the positive feedback loop has been published (Shapiro and Zhang 2001). In this feedback loop, SA levels potentiate the response to PCD "triggering factors" produced as a direct consequence of $a v r / R$ gene-dependent molecular recognition of pathogen. In support of this model, only interactions in which initial HR triggering signals are weak, resulting in comparatively slow HR progression (Ritter and Dangl 1996; Shapiro and Zhang 2001), show strong SA dependence of the HR (Rate et al. 1999; Shapiro and Zhang 2001) and consequent SA dependence of downstream gene expression and systemic acquired resistance (Shapiro and Zhang 2001; Tao et al. 2003; Zhang and Shapiro 2002).

Data presented in this paper strongly support the existence of two additional feedback loops. In agreement with Rate and Greenberg (2001), measurement of ion leakage correlated with the HR implied a greater final extent of PCD in nprl mutant plants as compared with Columbia wild type (Fig. 1 and Table 2). This NPRI-dependent negative feedback loop controlling PCD is indicated in Figure 5 with a heavier weight line that terminates in a dash-head and is interrupted by the nprl mutation. However, this feedback loop cannot be the sole negative regulation of PCD. Greater ion leakage at late timepoints was seen in $n d r 1-1$ mutant plants or $n d r 1-1 / n p r 1-2$ double-mutant plants than in either nprl-2 single mutant plants or wild-type plants. These differences correlated with differences in the extent of leaf collapse. As the only proven direct effect of the ndrl-1 mutation is the interruption of reactive oxygen-dependent upregulation of SA biosynthesis (Shapiro and Zhang 2001), it is reasonable to assume that this additional negative feedback pathway is SA-dependent.

Data presented in Figure 3 provided an important clue as to the nature of this second negative feedback loop. These data showed that in response to DC3000 $a v r B$, the final extent of increase in $\mathrm{H}_{2} \mathrm{O}_{2}$ levels in $n d r l-1$ or double-mutant plants was approximately half that seen with $n p r l-2$ or wild-type Columbia plants. Furthermore, the onset of accumulation was delayed slightly in these lines. We have argued that high-level reactive oxygen accumulation was a consequence of PCD, based on kinetic analysis and on logic stemming from analysis of the signaling block imposed by the ndrl-1 mutation (Shapiro and Zhang 2001). The kinetic analyses presented (Figs. 1 and 3) and microscopy-based measurements of time of onset of PCD (Zhang et al. 2003) were completely consistent with previous results. The correlation of enhanced PCD with reduced accumulation of $\mathrm{H}_{2} \mathrm{O}_{2}$ is thus counterintuitive.

The answer to this paradox most likely lies in the role of superoxide dismutase (SOD). Kinetic partitioning between dismutation and other fates (oxidation or reduction by alternative oxidants or reductants) results in some destruction of superoxide without consequent production of $\mathrm{H}_{2} \mathrm{O}_{2}$. Because of this competition, raising SOD levels can increase yield of $\mathrm{H}_{2} \mathrm{O}_{2}$ (Able et al. 2000). SOD induction in response to avirulent bacteria has been described (Epple et al. 2003; Kliebenstein et al. 1999). If the $n d r 1-1$ mutation impaired pathogen-induced increases in SOD levels, the result would be the observed decrease in final extent of (and slight delay in) $\mathrm{H}_{2} \mathrm{O}_{2}$ accumulation. Moreover, enhanced persistence of superoxide in the apoplast in the absence of SOD induction could explain the increased PCD seen at late timepoints in the HR with $n d r l-1$ and $n d r 1-1 / n p r 1-2$ plants.

Intriguingly, nprl-2 mutant plants were markedly impaired in SA accumulation $4 \mathrm{~h}$ postinoculation with DC3000•avrRpt2 and impaired at late timepoints postinoculation with 
DC3000•avrB. This impairment would not appear to be predicted by the placement of the nprl mutant block in signaling as shown in Figure 5. The likely explanation is that dead cells don't make SA. Using a microscopy-based assay, no PCD was observed $4 \mathrm{~h}$ postinoculation of Columbia plants with DC3000•avrRpt2 (Zhang et al. 2003). Consistent with these data, little if any increase in ion leakage was seen in this time interval (Fig. 1A). In nprl-2 plants, a small population of cells likely died slightly earlier than the corresponding population in wild-type plants due to impairment in NPRl-dependent negative feedback on PCD. If so, and if these cells were responsible for some of the SA biosynthesis, the earlier death of these cells would account for impaired SA accumulation at this timepoint. Small changes in extent of PCD at this timepoint would not have been observable in ion leakage-based experiments due to experimental noise (Fig. 1) and observed asynchrony in time of onset of PCD between replicate experiments (Zhang et al. 2003).

Results with DC3000•avrB provided further support for this hypothesis. In response to this strain, the rate of HR progression was much faster than with DC3000•avrRpt2 (Grant et al. 2000; Ritter and Dangl 1996; Shapiro and Zhang 2001). By the 6- to 7-h timepoints, much of the PCD that will occur in the HR has happened (Zhang et al. 2003). Differences between nprl-2 and wild-type plants in the extent of ion leakage were seen at these timepoints (Fig. 1C). The two- to threefold reduction in SA accumulation seen in nprl plants when compared with wild-type plants at late timepoints likely reflects a reduction in the number of cells remaining alive to make SA. Hence, the likely cellular compartmentation of SA synthesis can explain all observed anomalies in SA accumulation in nprl-2 plants.

The working model presented in Figure 5 is obviously an oversimplification. It is known, for example, that a second pathway also contributes to SA biosynthesis (Ferrari et al. 2003; Shapiro and Gutsche 2003; Wildermuth et al. 2001). The precise relationships of the components shown to other likely contributing elements, such as calcium fluxes, nitric oxide production, and caspase-like protease activity, are at present unclear and, thus, not pictured. Calcium fluxes appear to act early in PCD triggering and may be an important component of one or more "triggering factors" (Grant et al. 2000). We have recently established that nitric oxide accumulates close to the time of the first PCD events in the HR and appears to function in cell-to-cell communication governing HR progression (Zhang et al. 2003). Despite omission of these and other, at present ambiguous, signaling details, the combined action of the three described feedback loops can account for all data presented. We have further validated these hypotheses by constructing a differential equations-based mathematical model of these pathways and by showing that in silico simulated data closely matched the experimental data presented in this paper (Agrawal et al. 2003). Testable hypotheses generated based on this model should further refine knowledge of HR progression. Detailed understanding of PCD control will be necessary to exploit the HR in engineering of disease resistance and to avoid deleterious consequences of misregulation of PCD.

\section{MATERIALS AND METHODS}

\section{Plant materials and bacterial strains.}

All materials used will be made available upon request. The Arabidopsis thaliana lines and Pseudomonas syringae strains used in this paper have all been described previously (Shapiro 2000; Zhang and Shapiro 2002). The $n d r 1-1$ mutant is a null allele (Century et al. 1997). Analysis under conditions that assessed strictly NPRI-dependent phenotypes allowed us to conclude that nprl-2 is either a null allele or a severe hypomorph (Zhang and Shapiro [2002] discusses why some previous data addressing this point with this mutant were not interpreted correctly). The ndrl-1/nprl-2 double-mutant line has been submitted to the Arabidopsis Biological Resource Center (accession number CS6355). Arabidopsis thaliana was grown in a multitier plant chamber walk-in chamber (Conviron, Winnipeg, Manitoba, Canada) set for $8 \mathrm{~h}$ of light, $16 \mathrm{~h}$ of darkness at $22^{\circ} \mathrm{C}$ with $70 \%$ relative humidity. No readily observable differences were noted in plant growth or development between the four plant lines used in this study. Clear, plastic domes were placed loosely on flats during experiments to reduce airflow experienced by the plants. HR progression was identical with or without a dome when measured with the conductivity-based assay (data not shown). However, the dome delayed macroscopic leaf collapse. This delay allowed later timepoints to be taken in $\mathrm{H}_{2} \mathrm{O}_{2}$ and $\mathrm{SA}$ quantitation experiments. Direct comparison of $\mathrm{H}_{2} \mathrm{O}_{2}$ accumulation under a dome in this chamber to that documented previously using a reach-in chamber with less airflow (no domes) (Shapiro and Zhang 2001) showed identical kinetics (data not shown).

\section{Assessment of HR by macroscopic leaf collapse.}

Macroscopic HR assessment was as previously described (Shapiro 2000). HR was assessed approximately $20 \mathrm{~h}$ postinoculation with strains based on Pseudomonas syringae pv. tomato DC3000 or P. syringae pv. maculicola ES4326. The level of inoculum used was $2 \times 10^{7}$ per $\mathrm{ml}\left(\mathrm{OD}_{600}=0.02\right)$. Strains based on $P$. syringae pv. phaseolicola strain 3121 or $P$. syringae pv. glycinea race 5 were each used at four different levels of inoculum $\left(1 \times 10^{8}, 2 \times 10^{8}, 4 \times 10^{8}\right.$, and $1 \times 10^{9}$ bacteria per $\mathrm{ml}$ ). The 15 replicate leaves used for each combination of plant line, bacterial strain, and level of inoculum came from plants grown on three separate occasions.

\section{Assessment of ion leakage \\ using conductivity measurements.}

A single inoculated plant was used for each timepoint. At the time of sampling, 10 samples $\left(0.125 \mathrm{~cm}^{2}\right)$ of inoculated leaf tissue were taken, using a 4-mm diameter punch. Care was taken to avoid the midrib and sites of inoculation. No more than two leaf samples were taken from single leaves. The samples were rinsed in MilliQ-purified water and were patted dry on a paper towel. The leaf disks were put into a $10-\mathrm{ml}$ syringe with $5 \mathrm{ml}$ of MilliQ-purified water. Samples were infiltrated with the water by covering the hole of the syringe with a gloved finger and applying a vacuum by depressing the plunger. Repetitive infiltration combined with manual shaking was performed for $3 \mathrm{~min}$. The syringe was left for an additional $10 \mathrm{~min}$. Liquid was then transferred to a tube, and the sample conductivity was measured, using a Traceable digital conductivity meter (VWR, West Chester, PA, U.S.A.). Leaf samples were then taken from the syringe and were combined with the liquid. Boiling for $30 \mathrm{~min}$ was followed by sample cooling overnight to room temperature. Conductivity was read in the morning to determine total releasable ions. Values for each timepoint were normalized to total releasable ions and averaged between five replicate experiments. An equation for sigmoidal kinetics (Michaud et al. 2003) was used for nonlinear, least squares fits to the data using KaleidaGraph software (Synergy Software, Reading, PA, U.S.A.).

$$
y=\left[b+(a-b) /\left(1+e^{-s \times(t-m)}\right)\right]
$$

Fit constants $: \mathrm{a}=$ curve amplitude, $\mathrm{b}=$ baseline, $\mathrm{s}=$ slope, $\mathrm{m}=$ midpoint

This treatment allowed separate comparisons of curve amplitude, baseline, slope, and midpoint. Student's $t$ tests were performed to determine significance of differences in each 
parameter between experiments using different plant lines. Values of $P$ were read from a $t$ table.

\section{$\mathrm{H}_{2} \mathrm{O}_{2}$ quantitation.}

DCFH-diacetate (DA) was dissolved in dimethyl sulfoxide to make a $30 \mathrm{mM}$ stock solution that was diluted 100 -fold in water immediately prior to use. Leaves were infiltrated with DCFH-DA through the same needle hole used to introduce the bacteria $15 \mathrm{~min}$ prior to harvest. Whole leaf images were taken using an Axioskop 2 upright microscope (Carl Zeiss MicroImaging, Inc., Thornwood, NY, U.S.A.) equipped with a 1.6 $\mathrm{M}^{2} \mathrm{BIO}$ lens and an Axiocam digital camera. Leaves were excised and immediately put directly under the lens for imaging. Excitation used a mercury lamp and a GFP470 excitation filter. Green fluorescence was detected and quantified using a GFP525 emission filter. Constant exposure time was used for all experiments. No dye photobleaching was observed following the single, rapid, low-magnification exposures of each leaf used. Average pixel intensities of three identically sized areas of the same leaf were measured using the Fovea Pro 3.0 image processing tool kit plug-in of Photoshop 6.0 (Adobe, San Jose, CA, U.S.A.). The area immediately surrounding a needle hole was avoided. Experiments were repeated three times, and the means of the averaged pixel intensities were calculated for each timepoint.

\section{Measurement of SA.}

Extraction of leaf phenolics and capillary electrophoresisbased separation and quantitation of total SA levels were as in previous work from our laboratory (Shapiro and Gutsche 2003).

\section{ACKNOWLEDGMENTS}

The authors thank K. J. Czymmek (University of Delaware) for advice on microscopy and M. Kruempelman, C. McEwen, and B. Clements (DuPont Central Research and Development) for use of their capillary electrophoresis apparatus. This research was supported by a grant from the United States Department of Agriculture (NRICGP grant no. 0001256) and a University of Delaware Research Foundation grant to A. D. Shapiro. C. Zhang was supported in part by a predoctoral research assistantship from the University of Delaware College of Agriculture and Natural Resources and by a University Competitive Fellowship.

\section{LITERATURE CITED}

Able, A. J., Guest, D. I., and Sutherland, M. W. 2000. Hydrogen Peroxide yields during the incompatible interaction of tobacco suspension cells inoculated with Phytophthora nicotianae. Plant Physiol. 124:899-910.

Agrawal, V., Zhang, C., Shapiro, A. D., and Dhurjati, P. S. 2003. A dynamic mathematical model to clarify signaling circuitry underlying programmed cell death control in Arabidopsis disease resistance. Biotechnol. Prog. In press.

Atkinson, M. M., Baker, C. J., and Collmer, A. 1986. Transient activation of plasmalemma $\mathrm{K}^{+}$efflux and $\mathrm{H}^{+}$influx in tobacco by a pectate lyase isozyme from Erwinia chrysanthemi. Plant Physiol. 82:142-146.

Atkinson, M. M., Keppler, L. D., Orlandi, E. W., Baker, C. J., and Mischke, C. F. 1990. Involvement of plasma membrane calcium influx in bacterial induction of the $\mathrm{K}^{+} / \mathrm{H}^{+}$and hypersensitive responses in tobacco. Plant Physiol. 92:215-221.

Axtell, M. J., and Staskawicz, B. J. 2003. Initiation of RPS2-specified disease resistance in Arabidopsis is coupled to the avrRpt2-directed elimination of RIN4. Cell 112:369-377.

Baker, C. J., Atkinson, M. M., and Collmer, A. 1987. Concurrent loss in Tn5 mutants of Pseudomonas syringae pv. syringae of the ability to induce the hypersensitive response and host plasma membrane $\mathrm{K}^{+} / \mathrm{H}^{+}$ exchange in tobacco. Phytopathology 77:1268-1272.

Casper-Lindley, C., Dahlbeck, D., Clark, E. T., and Staskawicz, B. J. 2002. Direct biochemical evidence for the type III secretion-dependent translocation of the AvrBs2 effector into plant cells. Proc. Natl. Acad. Sci. U.S.A. 99:8336-8341.

Century, K. S., Holub, E. B., and Staskawicz, B. J. 1995. NDR1, a locus of Arabidopsis thaliana that is required for disease resistance to both a bacterial and a fungal pathogen. Proc. Natl. Acad. Sci. U.S.A. 92:65976601

Century, K. S., Shapiro, A. D., Repetti, P. P., Dahlbeck, D., Holub, E., and Staskawicz, B. J. 1997. NDR1: A pathogen-induced component required for Arabidopsis disease resistance. Science 278:1963-1965.

Clarke, A., Desikan, R., Hurst, R. D., Hancock, J. T., and Neill, S. J. 2000. No way back: Nitric oxide and programmed cell death in Arabidopsis thaliana suspension cultures. Plant J. 24:667-677.

Clough, S. J., Fengler, K. A., Yu, I.-C., Lippok, B., Smith, R. K. J., and Bent, A. F. 2000. The Arabidopsis dndl "defense, no death" gene encodes a mutated cyclic nucleotide-gated ion channel. Proc. Natl. Acad. Sci. U.S.A. 97:9323-9328.

del Pozo, O., and Lam, E. 1998. Caspases and programmed cell death in the hypersensitive response of plants to pathogens. Curr. Biol. 8:1129-1132.

Delaney, T. P., Friedrich, L., and Ryals, J. A. 1995. Arabidopsis signal transduction mutant defective in chemically and biologically induced disease resistance. Proc. Natl. Acad. Sci. U.S.A. 92:6602-6606.

Delledonne, M., Xia, Y., Dixon, R. A., and Lamb, C. 1998. Nitric oxide functions as a signal in plant disease resistance. Nature 394:585-588.

Delledonne, M., Zeier, J., Marocco, A., and Lamb, C. 2001. Signal interactions between nitric oxide and reactive oxygen intermediates in the plant hypersensitive disease resistance response. Proc. Natl. Acad. Sci. U.S.A. 98:13454-13459.

Devadas, S. K., and Raina, R. 2002. Preexisting systemic acquired resistance suppresses hypersensitive response-associated cell death in Arabidopsis hrll mutant. Plant Physiol. 128:1234-1244.

Durner, J., Wendehenne, D., and Klessig, D. F. 1998. Defense gene induction in tobacco by nitric oxide, cyclic GMP, and cyclic ADP-ribose. Proc. Natl. Acad. Sci. U.S.A. 95:10328-10333.

Epple, P., Mack, A. A., Morris, V. R. F., and Dangl, J. L. 2003. Antagonistic control of oxidative stress-induced cell death in Arabidopsis by two related, plant-specific zinc finger proteins. Proc. Natl. Acad. Sci. U.S.A. 100:6831-6836.

Ferrari, S., Plotnikova, J. M., De Lorenzo, G., and Ausubel, F. M. 2003. Arabidopsis local resistance to Botrytis cinerea involves salicylic acid and camalexin and requires eds 4 and pad2, but not sid2, eds 5 or pad4. Plant J. 35:193-205.

Glazener, J. A., Orlandi, E. W., and Baker, C. J. 1996. The active oxygen response of cell suspensions to incompatible bacteria is not sufficient to cause hypersensitive cell death. Plant Physiol. 110:759-763.

Goodman, R. N., and Novacky, A. J. 1994. The Hypersensitive Response in Plants to Pathogens: A Resistance Phenomenon. American Phytopathological Society, St. Paul, MN, U.S.A.

Gopalan, S., Bauer, D. W., Alfano, J. R., Loniello, A. O., He, S. Y., and Collmer, A. 1996. Expression of the Pseudomonas syringae avirulence protein $a v r B$ in plant cells alleviates its dependence on the hypersensitive response and pathogenicity (Hrp) secretion system in genotypespecific hypersensitive cell death. Plant Cell 8:1095-1105.

Grant, M., Brown, I., Adams, S., Knight, M., Ainslie, A., and Mansfield, J. 2000. The RPM1 plant disease resistance gene facilitates a rapid and sustained increase in cytosolic calcium that is necessary for the oxidative burst and hypersensitive cell death. Plant J. 23:441-450.

Heath, M. C. 1998. Involvement of reactive oxygen species in the response of resistant (hypersensitive) or susceptible cowpeas to the cowpea rust fungus. New Phytol. 138:251-263.

Hu, G., Yalpani, N., Briggs, S. P., and Johal, G. S. 1998. A porphyrin pathway impairment is responsible for the phenotype of a dominant disease lesion mimic mutant of maize. Plant Cell 10:1095-1105.

Jabs, T., Dietrich, R. A., and Dangl, J. L. 1996. Initiation of runaway cell death in an Arabidopsis mutant by extracellular superoxide. Science 273:1853-1856.

Kachroo, P., Yoshioka, K., Shah, J., Dooner, H. K., and Klessig, D. F 2000. Resistance to Turnip crinkle virus in Arabidopsis is regulated by two host genes and is salicylic acid dependent but NPR1, ethylene, and jasmonate independent. Plant Cell 12:677-690.

Kliebenstein, D. J., Dietrich, R. A., Martin, A. C., Last, R. L., and Dangl, J. L. 1999. LSD1 regulates salicylic acid induction of copper zinc superoxide dismutase in Arabidopsis thaliana. Mol. Plant-Microbe Interact. 12:1022-1026.

Lam, E., and del Pozo, O. 2000. Caspase-like protease involvement in the control of plant cell death. Plant Mol. Biol. 44:417-428.

Lam, E., Kato, N., and Lawton, M. 2001. Programmed cell death, mitochondria and the plant hypersensitive response. Nature 411:848-853.

Leister, R. T., Ausubel, F. M., and Katagiri, F. 1996. Molecular recognition of pathogen attack occurs inside of plant cells in plant disease resistance specified by the Arabidopsis genes RPS2 and RPM1. Proc. Natl. Acad. Sci. U.S.A. 93:15497-15502.

Mackey, D., Holt, B. F., Wiig, A., and Dangl, J. L. 2002. RIN4 interacts with Pseudomonas syringae type III effector molecules and is required for RPM1-mediated resistance in Arabidopsis. Cell 108:743-754. 
Mackey, D., Belkhadir, Y., Alonso, J. M., Ecker, J. R., and Dangl, J. L. 2003. Arabidopsis RIN4 is a target of the type III virulence effector avrRpt2 and modulates RPS2-mediated resistance. Cell 112:379-389.

Mazel, A., and Levine, A. 2001. Induction of cell death in Arabidopsis by superoxide in combination with salicylic acid or with protein synthesis inhibitors. Free Radic. Biol. Med. 30:98-106.

McDonald, K. L., Sutherland, M. W., and Guest, D. I. 2002. Temporary hypoxia suppresses the oxidative burst and subsequent hypersensitive cell death in cells of tobacco and soybean challenged with zoospores of incompatible isolates of Phytophthora species. Physiol. Mol. Plant Path. 61:133-140.

Michaud, D. J., Marsh, A. G., and Dhurjati, P. S. 2003. eXPatGen: Generating dynamic expression patterns for the systematic evaluation of analytical methods. Bioinformatics 19:1140-1146.

Mur, L. A. J., Bi, Y.-M., Darby, R. M., Firek, S., and Draper, J. 1997. Compromising early salicylic acid accumulation delays the hypersensitive response and increases viral disperal during lesion establishment in TMV-infected tobacco. Plant J. 12:1113-1126.

Mur, L. A. J., Brown, I. R., Darby, R. M., Bestwick, C. S., Bi, Y.-M., Mansfield, J. W., and Draper, J. 2000. A loss of resistance to avirulent bacterial pathogens in tobacco is associated with the attenuation of a salicylic acid-potentiated oxidative burst. Plant J. 23:609-621.

Nawrath, C., and Métraux, J. P. 1999. Salicylic acid induction-deficient mutants of Arabidopsis express PR-2 and PR-5 and accumulate high levels of camalexin after pathogen inoculation. Plant Cell 11:13931404.

Orlandi, E. W., Hutcheson, S. W., and Baker, C. J. 1992. Early physiological responses associated with race-specific recognition in soybean leaf tissue and cell suspensions treated with Pseudomonas syringae pv. glycinea. Physiol. Mol. Plant Path. 40:173-180.

Pirhonen, M. U., Lidell, M. C., Rowley, D. L., Lee, S. W., Jin, S. M. Liang, Y. Q., Silverstone, S., Keen, N. T., and Hutcheson, S. W. 1996. Phenotypic expression of Pseudomonas syringae avr genes in E. coli is linked to the activities of the $h r p$-encoded secretion system. Mol. PlantMicrobe Interact. 9:252-260.

Rate, D. N., and Greenberg, J. T. 2001. The Arabidopsis aberrant growth and death 2 mutant shows resistance to Pseudomonas syringae and reveals a role for NPR1 in suppressing hypersensitive cell death. Plant J. 27:203-211.

Rate, D. N., Cuenca, J. V., Bowman, G. R., Guttman, D. S., and Greenberg, J. T. 1999. The gain-of-function Arabidopsis acd6 mutant reveals novel regulation and function of the salicylic acid signaling pathway in controlling cell death, defenses, and cell growth. Plant Cell 11:1695-1708.

Ritter, C., and Dangl, J. L. 1996. Interference between two specific pathogen recognition events mediated by distinct plant disease resistance genes. Plant Cell 8:251-257.

Shah, J., Tsui, F., and Klessig, D. F. 1997. Characterization of a salicylic acid-insensitive mutant (sail) of Arabidopsis thaliana, identified in a selective screen utilizing the SA-inducible expression of the tms 2 gene.
Mol. Plant-Microbe Interact. 10:69-78.

Shapiro, A. D. 2000. Using Arabidopsis mutants to delineate disease resistance signaling pathways. Can. J. Plant Pathol. 22:199-216.

Shapiro, A. D., and Gutsche, A. T. 2003. Capillary electrophoresis-based profiling and quantitation of total salicylic acid and related phenolics for analysis of early signaling in Arabidopsis disease resistance. Anal Biochem. 320:223-233.

Shapiro, A. D., and Zhang, C. 2001. The role of NDRl in avirulence genedirected signaling and control of programmed cell death in Arabidopsis. Plant Physiol. 127:1089-1101.

Shirasu, K., Nakajima, H., Rajasekhar, V. K., Dixon, R. A., and Lamb, C. 1997. Salicylic acid potentiates an agonist-dependent gain control that amplifies pathogen signals in the activation of defense mechanisms. Plant Cell 9:261-270.

Takahashi, A., Kawasaki, T., Henmi, K., Shii, K., Kodama, O., Satoh, H. and Shimamoto, K. 1999. Lesion mimic mutants of rice with alterations in early events of defense. Plant J. 17:535-545.

Tao, Y., Xie, Z., Chen, W., Glazebrook, J., Chang, H.-S., Han, B., Zhu, T. Zou, G., and Katagiri, F. 2003. Quantitative nature of Arabidopsis responses during compatible and incompatible interactions with the bacterial pathogen Pseudomonas syringae. Plant Cell 15:317-330.

Torres, M. A., Dangl, J. L., and Jones, J. D. G. 2002. Arabidopsis gp91 ${ }^{\text {phox }}$ homologues AtrbohD and AtrBohF are required for accumulation of reactive oxygen intermediates in the plant defense response. Proc. Natl. Acad. Sci. U.S.A. 99:523-528.

Weymann, K., Hunt, M., Uknes, S., Neuenschwander, U., Lawton, K., Steiner, H.-Y., and Ryals, J. 1995. Suppression and restoration of lesion formation in Arabidopsis $l s d$ mutants. Plant Cell 7:2013-2022.

Wildermuth, M. C., Dewdney, J., Wu, G., and Ausubel, F. M. 2001. Isochorismate synthase is required to synthesize salicylic acid for plant defense. Nature 414:562-565.

Wolfe, J., Hutcheon, C. J., Higgins, V. J., and Cameron, R. K. 2000. A functional gene-for-gene interaction is required for the production of an oxidative burst in response to infection with avirulent Pseudomonas syringae pv. tomato in Arabidopsis thaliana. Physiol. Mol. Plant Path. 56:253-261.

Wu, Y., Wood, M. D., and Katagiri, F. 2003. Direct delivery of bacterial avirulence proteins into resistant Arabidopsis protoplasts leads to hypersensitive cell death. Plant J. 33:131-137.

Xu, H. X., and Heath, M. C. 1998. Role of calcium in signal transduction during the hypersensitive response caused by basidiospore-derived infection of the cowpea rust fungus. Plant Cell 10:585-597.

Zhang, C., and Shapiro, A. D. 2002. Two pathways act in an additive rather than obligatorily synergistic fashion to induce systemic acquired resistance and $P R$ gene expression. BMC Plant Biology 2:9.

Zhang, C., Czymmek, K. J., and Shapiro, A. D. 2003. Nitric oxide does not trigger early programmed cell death events but may contribute to cell-to-cell signaling governing progression of the Arabidopsis hypersensitive response. Mol. Plant-Microbe Interact. 16:962-972. 